J. Urol. Urogynäkol. AT 2019 • 26:15-19 https://doi.org/10.1007/s41972-019-0062-6 Online publiziert: 19. Februar 2019 (c) Der/die Autor(en) 2019

\section{Einleitung}

„Urinary urgency“ ist als imperativer Harndrang definiert. Die „International Continence Society“ (ICS) definiert den Begriff wie folgt: „a sudden compelling desire to pass urine which is difficult to defer" [1]. Die Definition von „urgency“ ist heute noch diskutierbar, da eine signifikante Patientengruppe „urgency“ als konstant störend und nicht imperativ beschreibt. „Urgency“ beinhaltet auch eine subjektive Interpretation. Dies zeigt sich in der Praxis, in der Harndrang individuell unterschiedlich beschrieben (Druck, Spannung, Schmerz, Wärme usw.) und unterschiedlich emotional bewertet wird. Die Literatur zeigt eine Prävalenzrate von „urgency“ zwischen 10,8 und $17,4 \%$ [2].

In vielen Fällen führt die Krankheit zu Chronifizierung, sozialer Isolation und Depression, nicht zuletzt aufgrund einer Tabuisierung dieses Themas in der Gesellschaft, oft sogar bei der ärztlichen Anamnese.

\section{Pathophysiologie}

\section{Afferenzen der Harnblase}

Über die afferenten Nervenbahnen der Hinterstränge des Rückenmarks sowie die Bahnen des Tractus spinothalamicus gelangen sowohl die exterozeptiven (Schmerz, Temperatur, Berührung) als auch die propriozeptiven (Dehnungs-, Kontraktionszustand) Reize der Harnblase zum Thalamus und informieren über Blasenfüllung und Harndrang. Die durch die vermehrte Blasendehnung aktivierten afferenten Signale werden bereits intraspinal oder zerebral unterdrückt. Ab einem Füllungsvolumen

\title{
Orietta Dalpiaz
}

Universitätsklinik für Urologie, Medizinische Universität Graz, Graz, Österreich

\section{„Urgency“: Ursachen, Diagnostik und Therapie}

von etwa $150-250 \mathrm{ml}$ wird ein erstes Harndranggefühl registriert, das mit Erreichen der Blasenkapazität zwischen 350 und $450 \mathrm{ml}$ als starker Harndrang wahrgenommen wird. Über das zentral gelegene pontine Miktionszentrum kann durch willkürliche Hemmung des Miktionsreflexes die Detrusorkontraktion so lange unterdrückt werden, bis die äuBeren Umstände eine Blasenentleerung zulassen.

Bei den Afferenzen der Harnblase werden 2 Typen unterschieden, die sich strukturell und funktionell voneinander unterscheiden. Die myelinisierten $\mathrm{A} \delta$ Afferenzen vermitteln Informationen über den Füllungszustand und werden bei passiver Dehnung aktiviert [3] C-Fasern vermitteln Schmerz, ausgelöst beispielsweise durch hohen intravesikalen Druck, chemische Noxen oder akute Entzündung. Es gibt steigendes Interesse bezüglich der Aktivierung von C-Afferenzen, die eine Rolle in der Wahrnehmung von „urgency“ spielen könnten [4]. Grundsätzlich werden im afferenten System 2 Hauptmechanismen der Übertragung eines Reizes in neuronale Aktionspotenziale unterschieden. Afferenzen können entweder durch den mechanischen Stimulus direkt oder chemisch über vorherige Mediatorfreisetzung aus umgebenden nichtneuronalen Zellen erregt werden [5]. Afferenzen (besonders C-Fasern) können verschiedene Neurotransmitter synthetisieren und eine große Auswahl von Rezeptoren, wie z.B. der transiente RezeptorPotenzial-Kationenkanal der Unterfamilie V (für Vanilloid) TRPV1, TRP-Kanäle A1 („transient receptor potential channels"), muskarinische und purinergische Subtypen, exprimieren. Weiters können Afferenzen durch Mediatoren wie Pros- taglandin (PG), Serotonin, Adenosintriphosphat (ATP) und Histamine aktiviert werden, die auch bei Entzündung, Verletzung und Ischämie freigesetzt werden und zu imperativem Harndrang führen.

\section{Integrität des Urothels}

Es gibt Evidenz, dass das komplexe neuronale Netzwerk der Harnblase auch von nichtneuronalen Zellen, wie z.B. dem Urothel, beeinflusst werden kann [5]. Das Urothel unterliegt ständigen funktionellen und strukturellen Veränderungen, um die Barrierefunktion aufrechtzuerhalten. Die Integrität des Urothels ist durch einen komplexen Prozess von Zellmigration und -proliferation bestimmt. Verschiedene Studien haben bewiesen, dass Reize (wie z.B. Infektion oder Ischämie) zu Einschränkungen dieser Reparaturmechanismen führen und dadurch die urotheliale Barriere und ihre Signalfunktion gestört werden [6]. Veränderungen des Urothels und dessen epithelialer Integrität führen durch Freisetzung von neuroaktiven Substanzen zu einer Störung des neuronalen Netzwerks, wodurch es zu einer erhöhten Aktivierung der afferenten Nervenfasern und zu "urgency“ kommt.

\section{Urotheliale Zellinteraktion}

Das Urothel ist ein metabolisch aktives Gewebe, reagiert auf mechanische und chemische Reize und stellt mit den anderen Blasenwandschichten und dem angrenzenden Bindegewebe eine funktionelle Einheit dar. Die Entstehung chemisch-sensorischer Informationen zwischen Urothel und Afferenzen wird zunehmend erforscht. Die physiologi- 


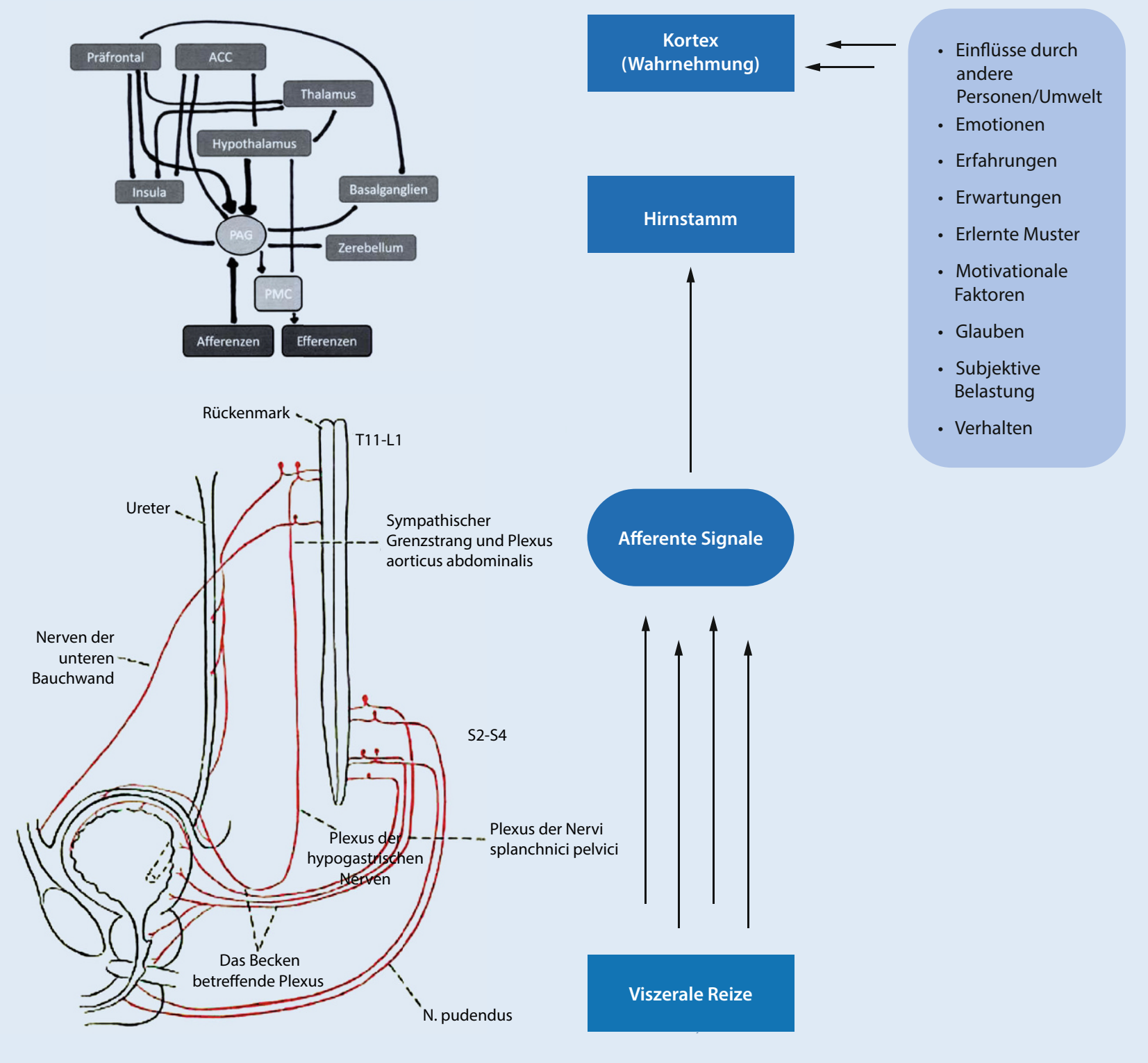

Abb. 1 ॥ Periphere und zentrale Steuerung:Koordination afferenter und efferenter Signale und Wahrnehmungsfaktoren des Harndranges. ACC Anteriorer cingulärer Cortex, PAG Periaquäduktales Grau, PMC Pontines Miktionszentrum

sche Dehnung der Harnblase sowie unterschiedliche Reize führen zur Sekretion von verschiedenen Mediatoren durch das Urothel, wie Azetylcholin, Prostaglandin, Serotonin, Adenosintriphosphat, Neurotrophinen und Zytokinen [5]. Ein Defekt dieser Signaltransduktion führt $\mathrm{zu}$ erhöhten afferenten Informationen und $\mathrm{zu}$ imperativem Harndrang.

\section{Zentrale Kontrolle}

Im zentralen Nervensystem werden die Koordination afferenter und efferenter
Signale gesteuert und die Wahrnehmung und Interpretation des Harndranges bestimmt. In den letzten Jahren konnten dank der Entwicklung der Neuroradiologie und durch funktionelle Magnetresonanztomographie (fMRT) verschiedene zuständige Gehirnregionen in der Füllungsphase der Blase beschrieben werden [7]. Im zentralen Nervensystem wird die Interpretation von Drang als normales oder imperatives Signal auch von emotionalen und kognitiven Faktoren beeinflusst (• Abb. 1).

\section{Klinik}

„Urgency“ ist ein Symptom, kommt als solches bei verschiedensten Pathologien vor und hängt auch vom Lebensstil ab (•Tab. 1). Die Symptome imperativer Harndrang, begleitet von Pollakisurie und Nykturie, mit oder ohne Dranginkontinenz, ohne Hinweis auf Harnwegsinfektionen oder andere Pathologie, wurden von der ICS als „overactive bladder“ (OAB) definiert [1]. „Urgency“ und OAB führen zu Veränderungen des Verhaltens (weniger trinken, häufige Toilettengänge, 
Einschränkung der Aktivitäten, sozialer Rückzug) und zu einer konsequenten Beeinträchtigung der Lebensqualität.

Die Wahrnehmung und die Interpretation von Harndrang als normales oder imperatives Signal werden von emotionalen und kognitiven Faktoren beeinflusst. Wird die Wahrnehmung von Drang mit Harnverlust, erlebten Situationen oder Schmerz verbunden, kann es zum erlernten Verhalten (z. B. Vermeiden bestimmter Orte, sozialer Rückzug) oder Angst und zur zentralen Chronifizierung des Symptoms kommen. Daher ist „urgen$c y$ " in der chronischen Form ähnlich zu behandeln wie andere Chronifizierungsprozesse.

\section{„Urgency“ im Alter}

Im Alter, bedingt durch Komorbiditäten (wie eingeschränkte zerebrale Leistungsfähigkeit, operative Eingriffe im kleinen Becken, subvesikale Obstruktion, Stoffwechselerkrankungen, neurologische Erkrankungen und Multimedikation), liegen multifaktorielle Ursachen für Harndrang vor (• Tab. 1). Weiters kommt es zu Detrusorveränderungen mit $\mathrm{Ab}$ nahme der elastischen Eigenschaften der Blasenwand, was als „,aging bladder“ bezeichnet wird. Die verminderte zentrale Hemmung des Miktionsreflexes führt aufgrund einer verspäteten Perzeption des Harndrangs und mangelhafter zentralmotorischer Hemmung des Miktionsreflexes zur zerebral enthemmten Blase mit den Symptomen der "urgency“ und OAB. Die mit dem Alter zunehmende Freisetzung von Neurotransmittern wie Azetylcholin aus nichtneuronalen Zellen des Urothels könnte im Zusammenhang mit der steigenden Prävalenz des OAB-Syndroms im Alter stehen.

\section{Diagnose}

Die Diagnose „urgency“ ist wegen der fehlenden Spezifität der Symptomatik eine Ausschlussdiagnose. Grunderkrankungen, die zum Drangsymptom führten, wie z. B. unspezifische Infektionen, anatomische Anomalien, subvesikale Obstruktion, Tumoren oder metabolische Störungen, müssen ausgeschlossen werden (- Tab. 1). Eventuelle Komorbi-

J. Urol. Urogynäkol. AT 2019 · 26:15-19 https://doi.org/10.1007/s41972-019-0062-6

(c) Der/die Autor(en) 2019

\section{O. Dalpiaz}

\section{"Urgency“: Ursachen, Diagnostik und Therapie}

\section{Zusammenfassung}

In der Urologie ist "urgency" als imperativer Harndrang definiert, welcher das Hauptsymptom des sog. "overactive bladder syndrome” $(\mathrm{OAB})$ darstellt und die Lebensqualität der Betroffenen stark beeinflusst. Die Definition des Harndrangs ist heute noch nicht einheitlich, da dieser auch eine subjektive Interpretation beinhaltet. Ein komplexes neuronales Netzwerk garantiert die Kontrolle des Füllungszustands der Harnblase und der Speicherung von Urin. Die Rolle des Urothels als metabolisch aktives Gewebe wird heute zunehmend beforscht. Veränderungen des Urothels und dessen epithelialer Integrität führen durch Freisetzung neuroaktiver
Substanzen zu einer Störung des neuronalen Netzwerks, wodurch es zu einer erhöhten Aktivierung der afferenten Nervenfasern und zu „urgency“ kommt.

Die Therapieoptionen reichen von Verhaltens-, Physio- und Pharmakotherapie über Neuromodulation bis zu operativen Maßnahmen. Diese Arbeit bietet eine Übersicht über die aktuellen pathophysiologischen Kenntnisse, Diagnostik und Therapieoptionen des imperativen Harndranges.

Schlüsselwörter

Afferenzen · Diagnose · Harndrang .

Überaktive Blase · Urothel

\section{Urinary urgency: causes, diagnosis, and treatment}

\section{Abstract}

Urinary urgency is defined as "a sudden compelling desire to pass urine which is difficult to defer". It is a highly prevalent symptom and is the key symptom of overactive bladder syndrome (OAB) that has a significant impact on quality of life. Sensory signaling in the urinary bladder has been traditionally attributed to direct activation of bladder afferents. There is evidence that the sensory system can be influenced by non-neuronal cells, such as the urothelium, which are able to respond to various stimuli releasing a variety of transmitters and mediators. The clinical assessment of urinary urgency represents a challenge: urgency is a symptom which remains difficult to define

ditäten oder Chronifizierungsfaktoren müssen bei der Anamnese erfasst werden.

Eine weitere Voraussetzung für die Diagnosestellung ist das Erfassen des Miktionsverhaltens der Betroffenen. Dazu dienen Miktionstagebuch oder Miktionsprotokoll, wobei die Trinkzeiten und -mengen sowie Blasenentleerungszeiten und die Urinmenge täglich notiert werden. Zur Messung des Schweregrads der "urgency“ stehen standardisierte validierte Fragebögen, wie die „Indevus urgency severity scale" (IUSS), die „urgency perception scale“ (UPS), die „urgency rating scale“(URS), die „patient and measure because it implies a subjective interpretation. Current first line treatment for urgency is behavioral and pharmacologic, but many patients remain dissatisfied with conservative measures. Advanced therapies, such as neuromodulation and botulinum toxin, can be employed. This paper presents an overview of the current knowledge about urgency relevant to clinicians and urologists with a focus on pathophysiology, evaluation and treatment.

Keywords

Bladder afferents · Diagnosis · Overactive bladder - Urinary urgency · Urothelium 
Tab. 1 Symptomvorkommen „urgency“

Lebensstil (Koffein, Alkohol, Trinkmenge)

Medikamente (Diuretika, Östrogene, Neuroleptika)

Erkrankungen des Harntrakts (Infektion, Blasentumoren, Harnsteine, Prostatahyperplasie, Strikturen)

Neurologische Erkrankungen (M. Parkinson, multiple Sklerose, Schlaganfall)

Internistische Erkrankungen (Diabetes, Herzinfarkt, COPD, Divertikulitis)

Muskuloskelettale Erkrankungen und Schmerzsyndrome

Voroperationen (Prostata, Inkontinenzoperationen/Sling, Ureterkatheter)

Radiotherapie

Psychische und psychiatrische Erkrankungen

\section{Therapie}

Die richtige Diagnose ist die Voraussetzung für eine erfolgreiche Therapie. Eine Verhaltenstherapie im Sinne einer Änderung des persönlichen Lebensstils, der Wahrnehmung der Harnblase und des Miktionsverhaltens sollte bei jedem Betroffenen eingeleitet werden. In der Behandlung der chronifizierten „urgency“ müssen Maßnahmen zur Beeinflussung des Erlebens und Verhaltens, der kognitiven Verknüpfung der Drangwahrnehmung und der daraus resultierenden subjektiven Bewertung von Drang einbezogen werden. Auch die Erwartungen der Betroffenen müssen berücksichtigt werden. Die Physiotherapie sollte auch als primäre Therapie eingesetzt werden. In einem aktuellen Review wurde die Wirksamkeit des Beckenbodentrainings in der Verbesserung der OAB-Symptome untersucht. Trotz unschlüssiger klinischer Daten zeigte sich eine nachweisliche Verbesserung der Lebensqualität [9]. Die „6th International Consultation on Incontinence “ bestätigte folgende Präparate (Grad A Level 1) für die Behandlung der „urgency“ und der OAB-Syndrome, und zwar: Darifenacin, Fesoterodin, Imidafenacin, Propiverin, Solifenacin, Tolterodin, Trospium und Oxybutynin [10]. Nach traditioneller Sichtweise bewirken Antimuskarinika eine Hemmung der willkürlichen und unwillkürlichen Detrusorkontraktion, wodurch auch eine Steigerung der funk- tionellen Blasenkapazität erreicht wird. Im Jahr 2012 wurden $\beta 3$-Agonisten, wie Mirabegron, von der „Food and Drug Administration" (FDA) zur Behandlung der Symptome von imperativem Harndrang und OAB zugelassen. Die Aktivierung der $\beta 3$-Rezeptoren bewirkt eine Detrusorrelaxation und somit eine Begünstigung des Speichervermögens der Blase. Viele Studien zeigten, dass Betroffene unter Mirabegronbehandlung eine bessere Compliance als bei einer Therapie mit Anticholinergika haben, da diese häufiger zu belastenden Nebenwirkungen führen. Das Ansprechen auf einzelne Präparate ist sehr individuell, sodass durchaus ein probatorisches Wechseln innerhalb einer Substanzgruppe oder eine Kombination von Präparaten sinnvoll sind. Die Studien Symphony, BESIDE und SYNERGY zeigten eine signifikante Besserung der Symptome und der Lebensqualität unter einer Kombinations- im Vergleich zur Monotherapie [11, 12]. Auch in der medikamentösen Therapie spielen individuelle Aspekte sowie das subjektive Empfinden und die Erwartungen der Betroffenen eine wichtige Rolle. Das erklärt das unterschiedliche Ansprechen und die Abbruchrate. Mit dem Ziel, die Aktivität des Detrusors zu verringern, zeigt auch die intravesikale Anwendung von Botulinumtoxin A eine signifikante und klinisch relevante Besserung der Drangsymptomatik [13].

Neuromodulationsverfahren wie die transkutane elektrische Nervenstimulation (TENS), die perkutane/transkutane tibiale Nervenstimulation (PTNS/TTNS) und die sakrale Neuromodulation (SNM) stellen sich auch bei „urgency“ und OAB als vielversprechende Therapie dar [14]. Durch die Stimulation von peripheren Afferenzen kommt es zu einer Modulation von Rückenmarksreflexen und Gehirnzentren. Eine aktuelle Studie verglich die Gehirnaktivität vor und nach SNM bei OAB-Patienten und zeigte eine signifikante Reduktion der Aktivität in der Insula und im präfrontalen und anterioren cingulären Kortex nach SNM [15]. In etwa $2 / 3$ der Betroffenen mit therapierefraktärem OAB zeigen eine signifikante Symptomverbesserung nach SNM.
Zusammenfassend stehen uns heute viele Therapien in der Behandlung von Harndrang zur Verfügung. Subjektive Wahrnehmung, individuelle Interpretation und Erwartung des imperativen Harndranges sollten in die Diagnose und Therapie unserer Patienten miteinbezogen werden.

\section{Korrespondenzadresse

 \\ PD Dr. Orietta Dalpiaz \\ Universitätsklinik für Urologie, Medizinische Universität Graz Auenbruggerplatz 29 8036 Graz, Österreich orietta.dalpiaz@ medunigraz.at}

Funding. Open access funding provided by Medical University of Graz.

\section{Einhaltung ethischer Richtlinien}

Interessenkonflikt. O.Dalpiaz gibt an, dass kein Interessenkonflikt besteht.

Dieser Beitrag beinhaltet keine von der Autorin durchgeführten Studien an Menschen oder Tieren.

Open Access. Dieser Artikel wird unter der Creative Commons Namensnennung 4.0 International Lizenz (http://creativecommons.org/licenses/by/4.0/deed. de) veröffentlicht, welche die Nutzung, Vervielfältigung, Bearbeitung, Verbreitung und Wiedergabe in jeglichem Medium und Format erlaubt, sofern Sie den/die ursprünglichen Autor(en) und die Quelle ordnungsgemäßnennen, einen Linkzur Creative Commons Lizenz beifügen und angeben, ob Änderungen vorgenommen wurden.

Hinweis des Verlags. Der Verlag bleibt in Hinblick auf geografische Zuordnungen und Gebietsbezeichnungen in veröffentlichten Karten und Institutsadressen neutral.

\section{Literatur}

1. Abrams P, Cardozo L, Fall M et al (2002) The standardisation of terminology of lower urinary tract function: report from the Standardisation Sub-committee of the International Continence Society. Neurourol Urodyn 21:167-178

2. Potts JM, Payne CK (2018) Urinary urgency in the elderly. Gerontology 64:541-550

3. Griffiths D (2015) Neural control of micturition in humans: a working model. Nat Rev Urol 12(12):695-705

4. Nazif O, Teichman JM, Gebhart GF (2007) Neural upregulation in interstitial cystitis. Urology 69(4 Suppl):24-33

5. Birder LA (2013) Nervous network for lower urinary tract function. Int JUrol 20(1):4-12 
6. Birder LA, Ruggieri M, Takeda M et al (2012) How does the urothelium affect bladder function in health and disease? ICI-RS 2011. Neurourol Urodyn 31(3):293-299

7. Griffiths D, Tadic SD (2008) Bladder control, urgency, and urge incontinence: evidence from functional brain imaging. Neurourol Urodyn 27(6):466-743

8. Starkman JS, Dmochowski RR (2008) Urgency Assessment in the Evaluation of Overactive Bladder (OAB). Neurourol Urodyn 27:13-21

9. Monteiro S, Riccetto C, Araújo A (2018) Efficacy of pelvic floor muscle training in women with overactive bladder syndrome: a systematic review. Int Urogynecol J29(11):1565-1573

10. Andersson KECL, Cruz F, Lee KS et al (2017) Pharmacological treatment of urinary incontinence. In: Abrams P, Cardozo L, Wagg A, Wein A (Hrsg) Incontinence, 6 . Aufl. International Continence Society, Bristol

11. Andersson KE, Choudhury N, Cornu JN et al (2018) The efficacy of mirabegron in the treatment of urgency and the potential utility of combination therapy. Ther Adv Urol 10(8):243-256

12. Drake MJ, Chapple C, Esen AA et al (2016) Efficacy and safety of mirabegron add-on therapy to solifenacin in incontinent overactive bladder patients with an inadequate response to initial 4-week solifenacin monotherapy: a randomised double-blind multicentre phase 3B study (BESIDE). EurUrol 70:136-145

13. Drake MJ, Nitti VW, Ginsberg DA et al (2017) Comparative assessment of the efficacy of onabotulinumtoxin A and oral therapies (anticholinergics and mirabegron) for overactive bladder: a systematic review and network meta-analysis. BJU Int 120(5):611-622

14. Tubaro A, Puccini F, De Nunzio C (2015) The management of overactive bladder: percutaneous tibial nerve stimulation, sacral nerve stimulation, or botulinum toxin? Curr Opin Urol 25:305-310

15. Weissbart SJ, Bhavsar R, Rao H et al (2018) Specific changes in brain activity during urgency in women with overactive bladder after successful sacral neuromodulation: a functional magnetic resonance imaging study. JUrol 200(2):382-388

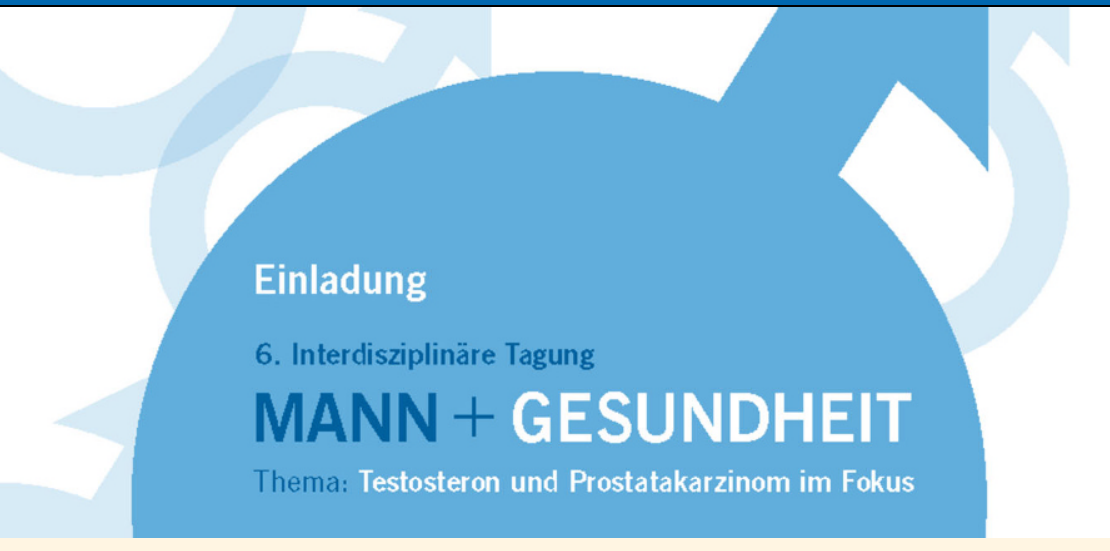

\section{Interdisziplinäre Tagung MANN + GESUNDHEIT}

Testosteron und Prostatakarzinom im Fokus

Samstag, 18. Mai 2019

09:00 bis 13:30 Uhr

Schlosspark Mauerbach

Herzog Friedrich-Platz 1, 3001 Mauerbach

Vorsitz: Prof. Dr. Michael Eisenmenger

Aus dem Programm:

\section{TESTOSTERON + ANDERE HORMONE \\ - Testosteron aus Sicht des Labormediziners \\ Univ.-Doz. Dr. Georg Endler \\ - Testosteron aus Sicht des Pharmazeuten \\ Mag. pharm. Andreas Gruber \\ - Stress und Hormone \\ Mag. pharm. Andreas Gruber \\ - Testosteron und Osteoporose \\ Prim. Univ.-Prof. Dr. Heinrich Resch \\ - Testosteron und das Androgen- \\ MetS}

Priv.-Doz. Dr. Markus Margreiter

\section{PROSTATAKARZINOM}

- Update Prostatakarzinom

Univ.-Prof. Dr. Gero Kramer

- Rehabilitation als Teil eines uroonkologischen Gesamtkonzeptes

Prim. Univ.-Prof. Dr. Alexander Gaiger

- PSA for Dummies

Prim. Univ.-Prof. Dr. Wolfgang Höltl

- Genetische Faktoren für Prognose und Therapie

OÄ Dr. Katharina Rötzer, PhD
Anrechenbarkeit der Veranstaltung

Eine DFP-Approbation ist vorgesehen.

\section{Kongressbüro/Anmeldung}

MEDahead Gesellschaft für medizinische

Information m.b.H.

Part of the Medical Opinion Network von MedMedia Verlag

Miriam Herbst

Tel.: 01/607 02 33-86 | Fax: 01/607 02 33-986

m.herbst@medahead.at

\section{Online-Anmeldung}

www.medahead-fortbildung.at/-

event/mann-gesundheit-2019

\section{Anmeldung bis 15. April 2019}

Diese Veranstaltung ist nur für medizinisches Fachpersonal.

Bitte geben Sie bei der Anmeldung Ihre Ärztekammernummer bekannt.

\section{Eintritt frei}

Ärztlicher Fortbildungsanbieter/ Wissenschaftliche Leitung

Österreichische Gesellschaft für Mann und Gesundheit

www.mann-und-gesundheit.at

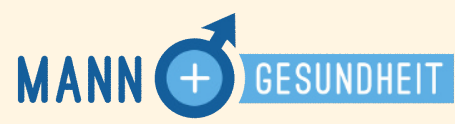

